\title{
Farmacología en la nutrición
}

\author{
Pharmacology in nutrition
}

Ana M. Pérez - Garza ${ }^{a}$, José F. Hernández Gracia ${ }^{b}$

\begin{abstract}
:
It is very important to consider the relationship that exists between nutrition and pharmacology, nowadays the new procedures of nutrition contemplate the use of different drugs that allow a better functioning in the treatment of a person. Therefore, in the area of nutrition is very important to know the concepts and definitions of pharmacology, in such a way that it allows to understand its operation and its relationship with nutrition for better use.
\end{abstract}

Keywords:

Nutrition, Pharmacology

\section{Resumen:}

Es muy importante considerar la relación que existe entre la nutrición y la farmacología, hoy en día los nuevos procedimientos de la nutrición contemplan el uso de diferentes fármacos que permiten un mejor funcionamiento en el tratamiento de una persona. Por lo anterior, en el área de nutrición es de suma importancia conocer los conceptos y definiciones de la farmacología, de tal manera que permita entender su funcionamiento y su relación con la nutrición para un mejor aprovechamiento.

\section{Palabras Clave:}

Nutrición, Farmacología

\section{Introducción}

El desarrollo del mapa conceptual nos permite reflexionar y retomar los conceptos y definiciones que estudiamos en las actividades anteriores, tales como la historia y evolución de la farmacología, también destaca en este trabajo la definición que me deja claro la función de la misma, así como sus ramas y la relación que existe con diferentes alimentos.

\footnotetext{
Autor de Correspondencia, Universidad Autónoma del Estado de Hidalgo, Escuela Superior de Atotonilco de Tula,, Email: anny.garza.73@gmail.com

${ }^{\mathrm{b}}$ Universidad Autónoma del Estado de Hidalgo, Escuela Superior de Atotonilco de Tula, ORCID: 0000-0001-5712-3018, Email: 


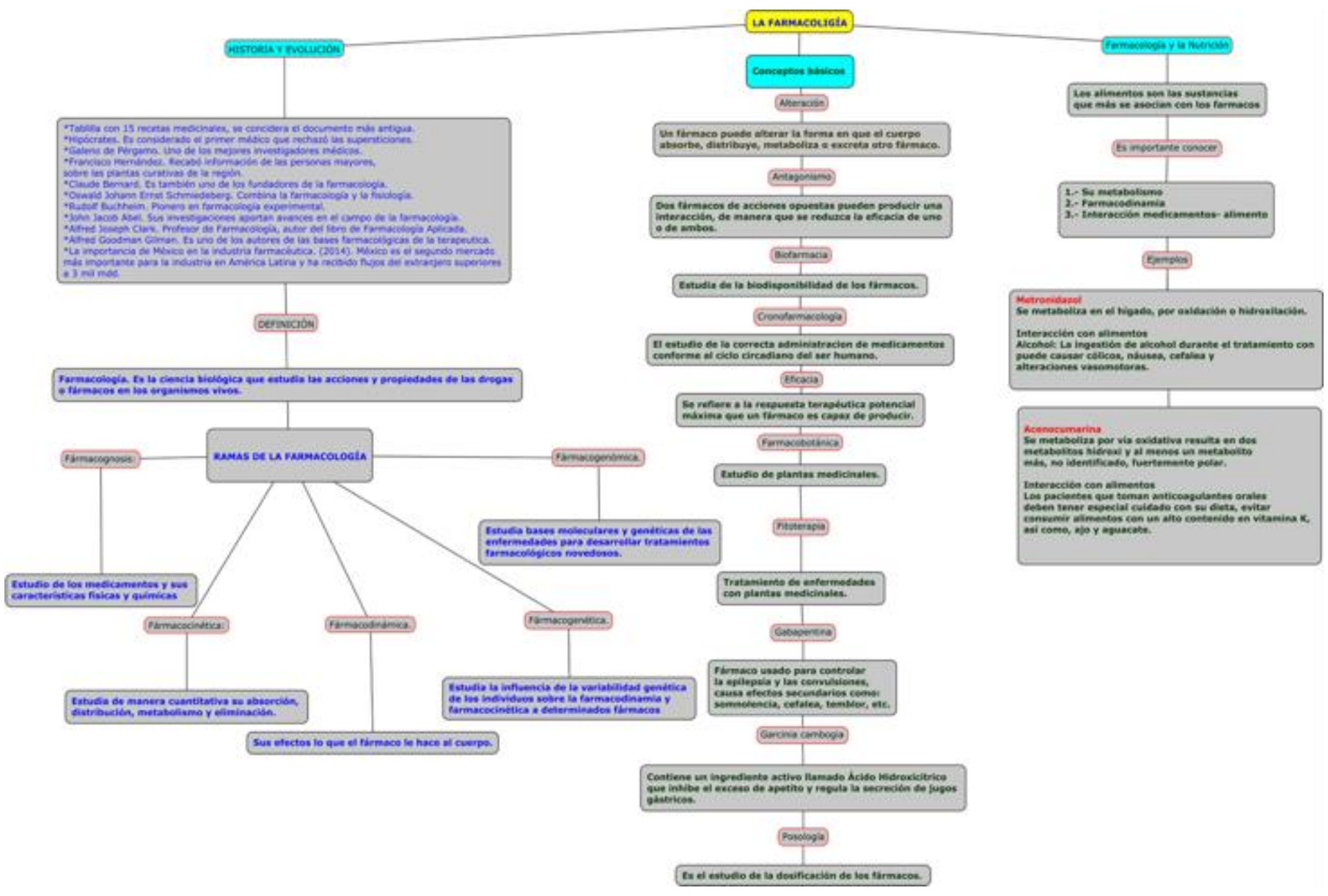

Figura 1. Conceptos y definiciones de la nutrición y la farmacología

\section{Conclusiones}

La relación de la farmacología con la nutrición es muy importante, hoy en día la tendencia es la ayuda de los fármacos como complemento en la evolución del paciente. Dos de los conceptos que me dejan claro la función de la farmacología es la Farmacocinética, que estudia de manera cuantitativa su absorción distribución, metabolismo y eliminación, y por otro lado la Farmacocinética que estudia los efectos, es decir lo que el fármaco hace al cuerpo. Por último, recalco la relación que hay entre la farmacología y la nutrición destacando su importancia ya que los alimentos son las sustancias que se relacionan con los fármacos.

\section{Referencias}

[1] Anticuagulado, (2015). Interacciones de los alimentos con los anticoagulantes. Recuperado de: https://anticoagulado.info/2012/09/18/interacciones-delos-alimentos-con-los-anticoagulantes/

[2] Fernández, I. (s.F.). Metronidazol. Recuperado de: http://www.infecto.edu.uy/terapeutica/atbfa/metro/METRONIDAZOL.htm

[3] García, N. (s.f.). Farmacología. Enfermería. Recuperado de: https://www.uv.mx/personal/lbotello/files/2016/03/FARMACODINAMIA-1.pdf

[4] Levy, M. (s.f.). Farmacología. Su Historia y desarrollo. Recuperado de: http://www.scf.sld.cu/PDF/farmacologia.pdf

[5] Novartis, (2018). Acenocumarol. Recuperado de: http://www.mufel.net/plm/prods/36203.htm

[6] Plata, L. (2013). Antecedentes y desarrollo Histórico de la farmacología. Recuperado de: https://es.slideshare.net/LizPlata/linea-del-tiempofarmacologia-26171955 
[7] Revista Medica, (2017). Historia de los medicamentos. Recuperado de: http://www.revistamedicina.net/anmdecolombia.net/images/documentos/Historia\%20de\%20los\%20Medicamentos\%203ra\%20edicion.pdf

[8] Salcedo, (2017). Campos de la Farmacología. SlidePlayer. Recuperado de: https://slideplayer.es/slide/11121790/

[9] San Miguel, M. T. \& Sánchez, J. L. (2011). Interacciones alimento/medicamento. Recuperado de: https://www.msssi.gob.es/biblioPublic/publicaciones/recursos_propios/infMedic/docs/vol35_1_Interacciones.pdf

[10] Unam, (2018). Metronidazol. Recuperado de: http://www.facmed.unam.mx/bmnd/gi_2k8/prods/PRODS/Metronidazol.htm

[11] Vademécum, (s.f.). Diclofenaco. Recuperado de: http://www.iqb.es/cbasicas/farma/farma04/d020.htm

[12] Vera, V. (2016). Glosario sobre algunos términos en farmacología. Silideshare. Recuperado de: https://es.slideshare.net/vincenzof28992/glosario-detrminos-en-farmacologa 